\title{
Efficacy and tolerability of sitagliptin and metformin compared with insulin as an initial therapy for newly diagnosed diabetic patients with severe hyperglycaemia
}

\author{
MINGQIAN HE ${ }^{1}$, MEI DENG ${ }^{2}$, JINGYA WANG $^{1}$, PENGXIA FAN $^{1}$, YUE WANG $^{1}$, \\ XINRUI ZHAO ${ }^{3}$, YIZHI HE ${ }^{4}$, BINGYIN SHI ${ }^{1}$ and JING SUI ${ }^{5}$

\begin{abstract}
${ }^{1}$ Department of Endocrinology, The First Affiliated Hospital of Xi'an JiaoTong University, Xi'an, Shaanxi 710061;
${ }^{2}$ Department of Endocrinology, Shanxian Central Hospital, Shanxian, Shandong 274300; ${ }^{3}$ Department of Immunology and

Rheumatology, The Second Affiliated Hospital of Xi'an JiaoTong University, Xi'an, Shaanxi 710004;

${ }^{4}$ Department of Endocrinology, Xi'an No. 3 Hospital, Xi'an, Shaanxi 710018; ${ }^{5}$ Department of Endocrinology and

International Medical Center, The First Affiliated Hospital of Xi'an JiaoTong University, Xi'an, Shaanxi 710061, P.R. China
\end{abstract}

Received April 14, 2020; Accepted December 8, 2020

DOI: $10.3892 /$ etm.2021.9649

\begin{abstract}
The safety and efficacy of dipeptidyl peptidase-4 inhibitors in patients newly diagnosed type 2 diabetes mellitus (T2DM) with severe hyperglycaemia have remained to be sufficiently demonstrated. The aim of the present study was to determine whether sitagliptin combined with metformin as an initial treatment had non-inferior outcomes with regards to glycaemic remission and $\beta$-cell function recovery to those of standard insulin therapy in this patient group. A prospective observational study was performed comparing the effects of sitagliptin combined with metformin and insulin therapy in a real-world clinical setting. A total of 168 participants were enrolled and received sitagliptin combined with metformin ( $\mathrm{Sig}$ ) or insulin (Ins) for almost 4 weeks. In addition, each group was further stratified into three subgroups, according to glycosylated haemoglobin (HbAlc) levels $(<10,10-12$ and $>12 \%)$. The primary outcomes were $\beta$-cell function and changes in fasting plasma glucose (FPG) and HbA1c at the 3-month follow-up.
\end{abstract}

Correspondence to: Dr Jing Sui, Department of Endocrinology and International Medical Center, The First Affiliated Hospital of Xi'an JiaoTong University, 277 West Yanta Road, Xi'an, Shaanxi 710061, P.R. China

E-mail: suijing1029@163.com

Abbreviations: ADA, American Diabetes Association; BMI, body mass index; T2DM, type 2 diabetes mellitus; DPP-4, dipeptidyl peptidase-4; GLP-1, glucagon-like peptide-1; FPG, fasting plasma glucose HbA1c, glycosylated haemoglobin; HOMA-IR, homeostasis model assessment of insulin resistance; Ins, insulin; AUC, area under the curve; MBCI, modified $\beta$-cell function index; OGTT, oral glucose tolerance test; Sig, sitagliptin; SMBG, self-monitored blood glucose; CI, confidence interval; $\mathrm{SD}$, standard deviation

Key words: sitagliptin, dipeptidyl peptidase-4 inhibitor, initial insulin therapy, severe hyperglycaemia, type 2 diabetes mellitus
Both insulin and sitagliptin combined with metformin reduced hyperglycaemia and achieved similar glycaemic outcomes, and no significant differences in FPG and HbAlc levels were obtained. No significant changes were observed in $\beta$-cell function concomitant with the glucose-lowering effects of the treatments. Of note, participants in the Ins group exhibited weight gain, whereas those in the Sig group had weight loss, with significant differences becoming evident after 1 month, particularly in the HbA1c $<10 \%$ subgroup. As compared with insulin injection, early treatment with sitagliptin combined with metformin in newly diagnosed patients with T2DM and severe hyperglycaemia produced non-inferior outcomes with regards to glycaemic remission. Therefore, combination of sitagliptin and metformin may be a viable initial treatment option for patients who prefer an alternative to insulin injection. This study was registered with ClinicalTrials.gov under no. NCT03180281.

\section{Introduction}

Type 2 diabetes mellitus (T2DM) is characterized by insulin resistance combined with progressive $\beta$-cell failure (1). Despite advances in the treatment of T2DM, devising an appropriate treatment strategy for patients with newly diagnosed T2DM with severe hyperglycaemia [glycosylated haemoglobin (HbA1c) $>9 \%$; fasting plasma glucose $(\mathrm{FPG}) \geq 11.1 \mathrm{mmol} / \mathrm{l}]$ provides a formidable challenge to physicians (2). Glucose toxicity and relative defects in insulin secretion make achieving glycaemic targets with metformin monotherapy difficult for these individuals (3). Therefore, patients with newly diagnosed severe hyperglycaemia require prompt and effective glycaemic control.

Most current guidelines, such as those by the American Diabetes Association (ADA) and American Association of Clinical Endocrinologists, indicate that either initial insulin treatment or oral antidiabetic drug treatment with metformin are options for patients with severe hyperglycaemia $(4,5)$. In addition, the ADA guidelines recommend that combination 
insulin treatment should be initiated when $\mathrm{HbA} 1 \mathrm{c}$ is at 286-108 $\mathrm{mmol} / \mathrm{mol}$ (10-12\%) (6). The ADA 2019 guidelines clearly mention that early introduction of insulin should be considered when $\mathrm{HbA1C}$ or blood glucose levels are high $[>10 \%(86 \mathrm{mmol} / \mathrm{mol})$ and $\geq 300 \mathrm{mg} / \mathrm{dl}(16.7 \mathrm{mmol} / \mathrm{l})$, respectively] (7). However, insulin treatment is associated with an increased risk of adverse events, such as high rates of severe hypoglycaemia, glycaemic variability and weight gain $(8,9)$. In addition, certain patients refuse insulin injections, partly due to fear of adverse effects, but also for other reasons (inconvenience, fear of injection and pain) (10). Therefore, an alternative should be developed for those patients.

Metformin, a classic drug used to treat T2DM, has been applied in clinical practice for decades, achieving acceptable therapeutic efficacy (11). It has a role in insulin resistance (hyperinsulinemia), which is closely related to the pathogenesis and mechanisms of T2DM (12). Sitagliptin, the first available dipeptidyl peptidase-4 (DPP-4) inhibitors, was approved as a treatment for T2DM by the US Food and Drug Administration in 2006, and it has been widely used in clinical practice. Safety and tolerability have been confirmed in clinical trials for up to 2 years, with a low risk of hypoglycaemia when administered as a monotherapy or when used in combination with antihyperglycaemic agents that are generally not known to cause hypoglycaemia (13). DPP-4 inhibitors are a novel group of medicines used for the treatment of T2DM, which improve meal stimulated insulin secretions by protecting glucagon-like peptide-1 (GLP-1) and glucose dependent insulinotropic polypeptide from enzymatic degradation (14). Sitagliptin is a well-tolerated monotherapy or add-on therapeutic agent used in combination with other oral antidiabetic agents; it has numerous favourable features, including once-daily administration, potent glucose-lowering effects, a low risk of hypoglycaemia and a neutral effect on body weight $(15,16)$.

However, an optimal treatment strategy for patients with T2DM with severe hyperglycaemia has remained to be provided. The present study aimed to prove that initiation therapy in patients with newly diagnosed T2DM presenting with severe hyperglycaemia should also include noninsulin alternatives, particularly for those patients who refuse to use insulin therapy. A prospective observational study was therefore performed to compare the efficacy and safety of sitagliptin combined with metformin vs. insulin as an initial treatment for patients newly diagnosed with T2DM with severe hyperglycaemia. In addition to glycaemic control, the effects of these treatments on insulin levels and $\beta$-cell insulin secretory capacity were investigated and an attempt was made to establish an optimal therapeutic regimen for these individuals.

\section{Materials and methods}

Study design. A prospective observational cohort study with a non-randomized design comparing sitagliptin combined with metformin and insulin therapy in patients with newly diagnosed T2DM with severe hyperglycaemia was performed. From January 2014 to June 2019, 168 consecutive participants from the First Affiliated Hospital of Xi'an JiaoTong University (Xi'an, China) in the northwest region of China, who had been newly diagnosed with T2DM according to the 1999 World
Health Organization diagnostic criteria, had severe hyperglycaemia [HbAlc $>9 \%$ and FPG $\geq 200 \mathrm{mg} / \mathrm{dl}$ (11.1 mmol/l)] and were symptomatic (present with hallmark symptoms of polyuria/polydipsia), were included in the study. An additional cohort of 448 patients with T2DM was enrolled at the outpatient service of the First Affiliated Hospital of Xi'an JiaoTong University (Xi'an, China). These patients were made to complete a Therapy Attitude Questionnaire to determine their preferred treatment and the specific reasons for their choice (10).

In the cohort of newly diagnosed patients, there were 2 treatment regimen options used based on the real-world settings. The option chosen was based on the clinician's choice, considering age, body mass index (BMI), economic conditions, other complications, HbAlc, fasting and postprandial plasma glucose of patients. A combination of sitagliptin (100 mg/day) and metformin (1,500 mg/day) in the Sig group, which were the recommended daily dosages and invariable (17). In the Ins group, three combinations of insulin therapy were used at the start of treatment: i) Insulin glargine or detemir combined with insulin for a total dosage of 0.3-0.4 U/ $\mathrm{kg} / \mathrm{day}$; ii) low-dose insulin glargine or detemir at a total dosage of 0.1-0.2 U/kg/day combined with oral hypoglycaemic agents, including metformin and acarbose, and excluding insulin secretagogues such as sulfonylureas and DPP-4 inhibitors; iii) Novolin 30R insulin at a total dosage of 0.3-0.4 U/ kg/day. The insulin regimens administered to the Ins group were regarded as a low dose, which is suitable for patients with newly diagnosed T2DM presenting with severe hyperglycaemia (17). FPG levels of $<7.0 \mathrm{mmol} / 1$ and postprandial blood glucose of $<10.0 \mathrm{mmol} / \mathrm{l}$ were considered to indicate euglycaemia. After achieving euglycaemia (almost within 4 weeks), sitagliptin and insulin therapy were paused, while lifestyle interventions and metformin therapy were continued. The experimental protocol was approved by the Ethics Committee of the First Affiliated Hospital of Xi'an JiaoTong University (Xi'an, China; approval no. XJTU1AF2016LSL-048). Informed consent was obtained from all participants.

Inclusion criteria. i) Males or females with newly diagnosed T2DM; ii) age, 18-70 years, FPG of $\geq 11.1 \mathrm{mmol} / 1$, HbA1c of $\geq 9.0 \%$ and $\mathrm{BMI}$ of $18-28 \mathrm{~kg} / \mathrm{m}^{2}$; iii) no previous treatment with antidiabetic or antihyperlipidaemic medication.

Exclusion criteria. i) T1DM, gestational diabetes or diabetes with an identifiable secondary cause; ii) evidence of elevated alanine or aspartate aminotransferase or significant renal impairment (estimated creatinine clearance of $<\mathrm{ml} / \mathrm{min}$ ); iii) severe complications associated with diabetes or severe infection; iv) history of organ transplantation, cancer, macrovascular disease, autonomic neuropathy, proliferative retinopathy, scheduled surgery or serious trauma; v) premenopausal females who were nursing, pregnant or with child-bearing potential.

Data collection. Anthropometric and laboratory data were collected prior to treatment and 1 and 3 months after treatment. An oral glucose tolerance test (OGTT) and insulin release test were conducted in a subgroup of participants 3 months after treatment and $\beta$-cell function was also 
evaluated (18). To calculate the homeostasis model assessment (HOMA) index, the following formulae were used: HOMA-insulin resistance $(I R)=$ fasting insulin (FINS) xFPG/22.5; HOMA- $\beta=20 x F I N S /(F P G-3.5)$ (18). The $\beta$-cell response to the OGTT was also calculated as the area under the curve (AUC) for plasma glucose (PG) and insulin (AUC-PG and AUC-INS) at 0, 60, 120 and 180 min using the trapezoid rule: $\mathrm{AUC}-\mathrm{PG}=\left(\mathrm{PG}_{0 \mathrm{~min}}+\mathrm{PG}_{60 \mathrm{~min}}\right) / 2+\mathrm{PG}_{120 \mathrm{~min}}+\mathrm{PG}_{180 \min }$; AUC-INS $=\left(\mathrm{INS}_{0 \min }+\mathrm{INS}_{60 \mathrm{~min}}\right) / 2+\mathrm{INS}_{120 \min }+\mathrm{INS}_{180 \min }$ (19); $\Delta \mathrm{I}_{60_{\text {min }}} / \Delta \mathrm{G} 60_{\text {min }}=\left(\mathrm{INS}_{60 \text { min }}-\mathrm{INS}_{0 \min }\right) /\left(\mathrm{PG}_{60 \text { min }}-\mathrm{PG}_{0 \min }\right), \mathrm{Ip} / \mathrm{I} 0=$ peak insulin/fasting insulin (20); disposition index $(\mathrm{DI})=\left(\Delta \mathrm{I} 60_{\min } / \Delta \mathrm{G} 60_{\mathrm{min}}\right) / \mathrm{HOMA}-\mathrm{IR} ;$ modified $\beta$-cell function index $(\mathrm{MBCI})=\left(\mathrm{INS}_{0 \min } \times \mathrm{PG}_{0 \min }\right) /\left(\mathrm{PG}_{120 \mathrm{~min}}+\mathrm{PG}_{60 \mathrm{~min}}{ }^{-}\right.$ $\left.2 \mathrm{xPG}_{0 \min }\right)(21)$.

Data regarding hypoglycaemic events were collected by asking participants and checking prescriptions. Hypoglycaemia was defined as self-monitored blood glucose (SMBG) of $<3.9 \mathrm{mmol} / \mathrm{l}$ with or without signs/symptoms of hypoglycaemia. Participants were asked about symptoms of hypoglycaemia that required medical or non-medical assistance, including heart palpitations, sweating, confusion and weakness or dizziness.

Follow-up. Outpatient examinations were performed prior to the beginning of the experiment and after 1 and 3 months. The 1-month visit was a safety evaluation, which included a physical examination, evaluation of glucose control (plasma FPG and SMBG recorded in the diary), reinforcement of lifestyle advice and completion of an adverse events record. An OGTT and insulin release test were performed in a subgroup of participants 3 months after treatment; demographic and biochemistry data were also collected.

Outcome measures. The primary endpoints were changes in FPG and HbAlc and assessment of islet $\beta$-cell function at the 3-month follow-up. Secondary endpoints included glycaemic remission rate, time of glycaemic remission, changes in body weight and BMI at the 1- and 3-month follow-ups, as well as hypoglycaemic episodes during the study period. Hypoglycaemia was assessed using a questionnaire and supplemented with SMBG values.

Data analysis and statistics. Noninferiority of the outcomes was assumed if the upper limit of the $95 \%$ confidence interval (CI) for the difference in the effects of the treatment was less than a $0.3 \%$ change in HbAlc levels from baseline to month 3. With the assumption of a standard deviation (SD) of $1.15 \%$, 49 patients in the Sig group and 98 patients in the Ins group (a total of 147 patients) were required to achieve 80\% power for the analysis.

All statistical analyses were performed using SPSS 22.0 software (IBM Corp.). Normally distributed data are reported as the mean \pm SD and non-normally distributed data as the median and interquartile range. Count data are expressed as $\mathrm{n}(\%)$. To test the differences between groups, a two-samples t-test was used if the normality criteria were met and Wilcoxon's rank-sum test if they were not. A paired t-test and repeated-measures ANOVA were used to evaluate differences after vs. prior to the intervention. For nominal parameters, differences among the groups were analysed using the $\chi^{2}$ test.
Subgroup analyses were conducted for each stratification factor (HbA1c at baseline $<10,10-12$ or $>12 \%$ ) in the two groups. A two-sided $\mathrm{P}<0.05$ was considered to indicate a statistically significant difference.

\section{Results}

Baseline clinical characteristics of the two treatment groups. Results of the Therapy Attitude Questionnaire, suggested that the additional cohort of 448 patients with T2DM, who had received numerous years of treatment for T2DM, preferred oral treatment and refused to use insulin injections (Table SI and Fig. S1). Of these, 56.92\% of all patients were male and $58.04 \%$ were more than 55 years old. $40.63 \%$ received oral treatment, $22.32 \%$ received both insulin and oral treatment and $37.05 \%$ received only insulin treatment (Table SI). However, only a small proportion of patients (7.81\%) preferred insulin as their treatment, while most patients with T2DM $(95.98 \%)$ rejected insulin therapy mainly due to inconvenience and fear of injection, adverse effects and pain (Fig. S1).

Furthermore, a total of 168 participants with newly diagnosed T2DM were recruited, including 64 in the Sig group and 104 in the Ins group. None of the participants dropped out and no serious adverse effects were observed during the intervention. The ADA guidelines recommend initiating combination insulin therapy at an HbAlc of $\geq 10-12 \%$ (86-108 $\mathrm{mmol} / \mathrm{mol}$ ) (2). Consequently, participants in each group were stratified into three subgroups according to their HbA1c levels. The baseline clinical characteristics of the participants (age, sex, weight and BMI) were similar and there were no statistically significant differences in glucose levels (HbA1c and FPG) between the two groups at the beginning of the study ( $\mathrm{P}>0.05$; Table I; the values for the total Ins and Sig groups are shown in Table SII).

Insulin as well as sitagliptin combined with metformin reduce hyperglycaemia in patients with newly diagnosed T2DM and severe hyperglycaemia. First, it was examined whether sitagliptin combined with metformin treatment was able to reduce hyperglycaemia in patients newly diagnosed T2DM with severe hyperglycaemia. Paired t-tests were used to evaluate differences in FPG and HbAlc after the intervention vs. baseline. FPG was significantly decreased after 1 month of intervention in both groups; thus, all participants had significantly improved glycaemic control and achieved euglycaemia within 1 month (all $\mathrm{P}<0.001$; Table II). At the 3-month follow-up, significant reductions in FPG and HbAlc were observed in these two groups as compared with the baseline values and euglycaemia was maintained (all $\mathrm{P}<0.001$; Table II).

Comparison of glycaemic outcome parameters following treatment. Following stratification into the three subgroups, no significant differences were observed in the baseline glucose levels (HbA1c and FPG; Table I) between the two groups and no significant differences were obtained in the changes in FPG ( $\triangle \mathrm{FPG})$ after the 1- or 3-month, and HbA1c ( $\triangle \mathrm{HbA} 1 \mathrm{c})$ after the 3-month follow-up in either group (all P>0.05; Table II).

Although both insulin and sitagliptin combined with metformin were able to reduce hyperglycaemia and achieve 


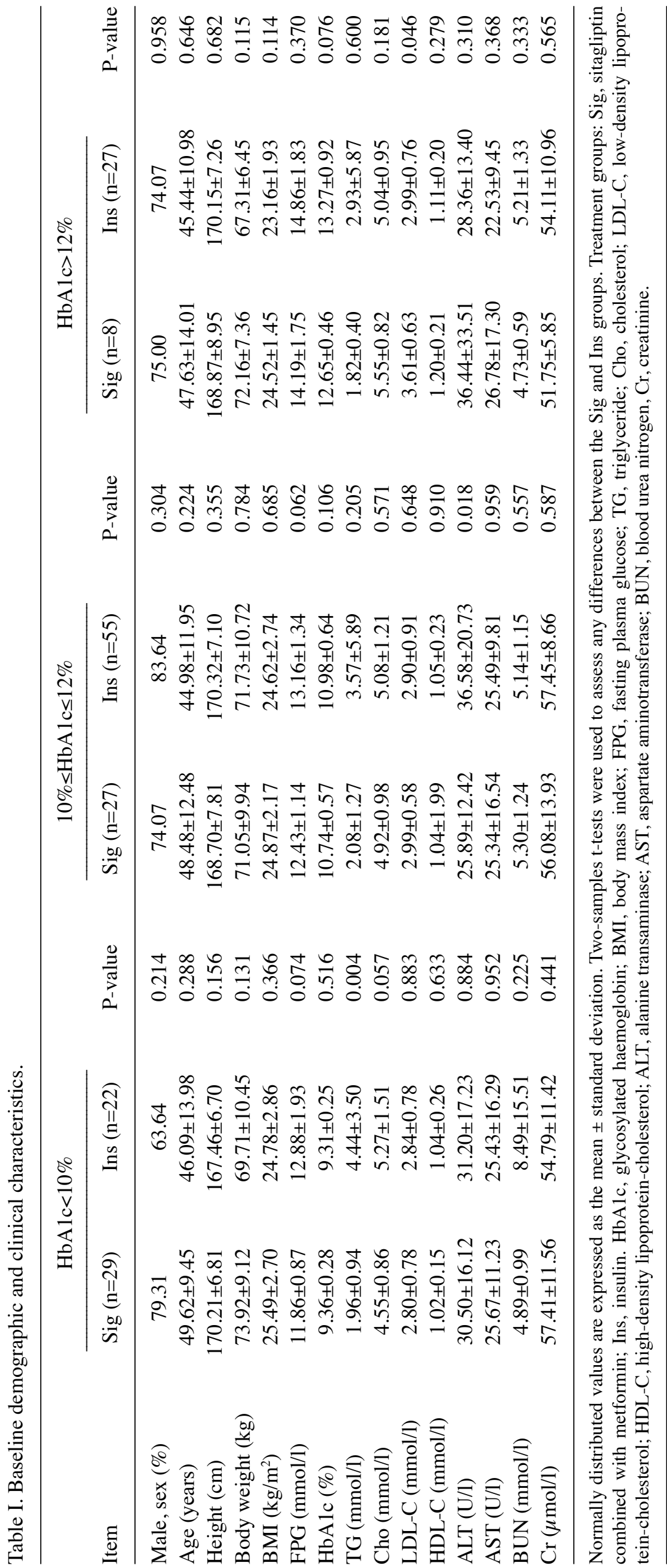




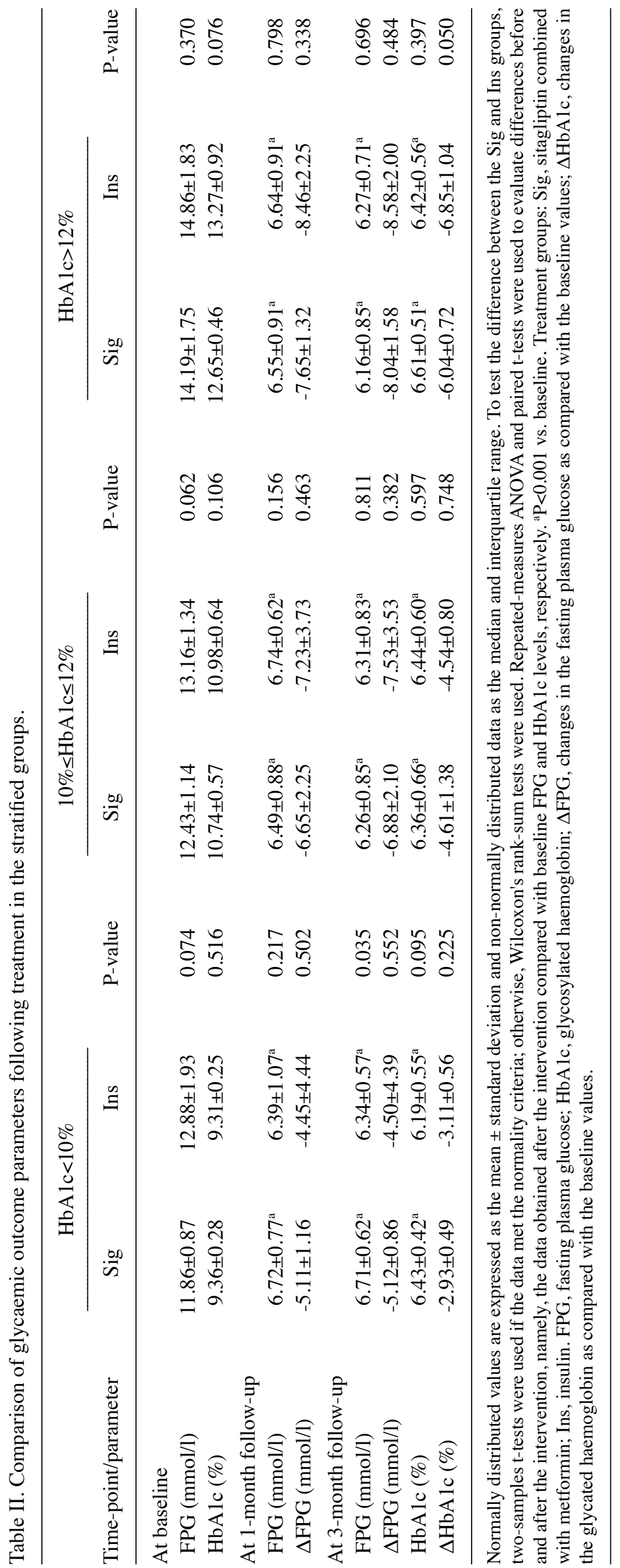


similar glycaemic outcomes, patients in the Ins group were able to achieve euglycaemia in a significantly shorter time than those in the $10 \% \geq \mathrm{HbA} 1 \mathrm{c} \leq 12 \%$ subgroup of the Sig group, with the average time to achieve euglycaemia being significantly shorter in the Ins than in the Sig group $(\mathrm{P}<0.001$; Fig 1$)$. The same trend was observed but no significant difference was observed in the other two subgroups ( $\mathrm{P}>0.05$; Fig. 1$)$.

Adverse events in the two groups following treatment. No severe hypoglycaemic events or other adverse events were reported in either group. Significant differences were observed in the changes in body weight between the Sig and Ins groups (Table III). Of note, participants in the Ins group exhibited body weight gain and those in the Sig group body-weight loss, with significant differences ( $\Delta$ body weight and $\Delta B M I$ between the two groups) becoming evident after 1 month. The mean change in BMI from baseline to 1 month was- $0.28 \pm 0.61 \mathrm{~kg} / \mathrm{m}^{2}$ in the Sig group and $0.26 \pm 0.36 \mathrm{~kg} / \mathrm{m}^{2}$ in the $\mathrm{HbA} 1 \mathrm{c}<10 \%$ subgroup of the Ins group ( $\mathrm{P}<0.001$; Table III). At the 3-month follow-up, those subgroups exhibited no significant differences in $\Delta$ body weight and $\Delta \mathrm{BMI}$ between the two groups ( $\mathrm{P}>0.05$; Table III).

In the present study, hypoglycaemia $[\leq 70 \mathrm{mg} / \mathrm{dl}$ (3.9 $\mathrm{mmol} / \mathrm{l})$ ] was reported by $6.25 \%$ of patients in the Sig group (4/64) and $6.73 \%$ of those in the Ins group (7/104), with no significant difference between groups (Table IV). No severe hypoglycaemia events were reported in the two groups.

Comparative effects of sitagliptin combined with metformin and insulin therapy on islet $\beta$-cell function. Glucose levels, total insulin response and amelioration of $\beta$-cell function between these two treatment groups were compared and no significant differences in HOMA-IR, HOMA- $\beta$, Ip/I0, $\Delta \mathrm{I} 60 / \Delta \mathrm{G} 60$, DI or MBCI were identified (all $\mathrm{P}>0.05$; Table $\mathrm{V}$ ). The glucose and insulin responses expressed as AUC (AUC-Ins, AUC-PG and AUC-PG/AUC-Ins) for a period of $180 \mathrm{~min}$ during the OGTT after 3 months of treatment were not significantly different between the two groups (all $\mathrm{P}>0.05$; Table V).

\section{Discussion}

Glucotoxicity in patients with newly diagnosed T2DM with severe hyperglycaemia presents considerable challenges for physicians in the quest to improve the current therapeutic regimen to meet individualized needs $(22,23)$. The present study assessed the use of the oral antidiabetic agent sitagliptin combined with metformin and insulin injection therapy in patients with newly diagnosed T2DM with severe hyperglycaemia by evaluating clinical efficacy, including the reduction in glucose excursions and differences in glucose amelioration and $\beta$-cell function.

Glucotoxicity was assessed and it was indicated that FPG decreased rapidly in both groups within the first month, even in severe glucotoxic states (HbA1c $>12 \%)$. Significant reductions in FPG and $\mathrm{HbA} 1 \mathrm{c}$ from baseline were observed in the two groups and euglycaemia was maintained after the 3-month follow-up, suggesting that both treatments were efficacious. However, the average intervention time to achieve euglycaemia in the Sig group was significantly longer than that in the $10 \% \leq \mathrm{HbA} 1 \mathrm{c} \leq 12 \%$ subgroup of the Ins group and more participants in the Ins group achieved the glycaemic control

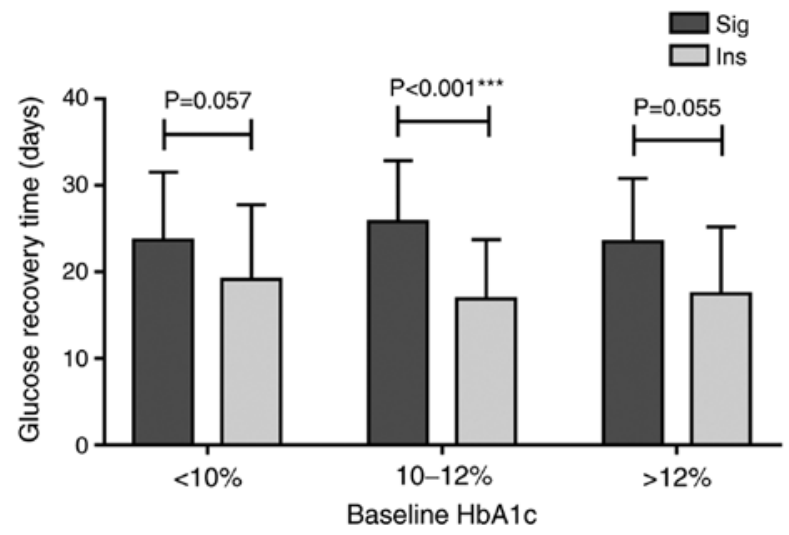

Figure 1. Comparison of average time to achieve euglycaemia in subgroups stratified by baseline HbA1c. ${ }^{* * *} \mathrm{P}<0.001$. Treatment groups: Sig, sitagliptin combined with metformin; Ins, insulin. HbA1c, glycosylated haemoglobin.

goal earlier than participants in the Sig group. The results were consistent with those of a previous study (24).

A patient-centred approach should be used to guide the choice of pharmacological agents. Considerations include efficacy, risk of hypoglycaemia, impact on body weight, potential side effects, cost and individual preferences (4). These possible adverse events may cause concern among patients with T2DM and physicians, possibly constituting major barriers to initiating and maintaining adherence to insulin treatment. The results obtained with the Therapy Attitude Questionnaire indicated that most patients preferred oral treatment and refused to receive insulin injections, mainly due to inconvenience, fear of injection, adverse effects and pain.

The UK Prospective Diabetes Study highlighted the importance of preserving $\beta$-cell function and insulin sensitivity in the management of T2DM $(25,26)$. Accumulating evidence suggests that DPP-4 inhibitors preserve pancreatic $\beta$-cell function $(25,27)$. In the real-world clinical setting, endocrinologists refuse to perform OGTT for newly diagnosed patients with T2DM and severe hyperglycaemia, since oral glucose administration to patients with glucotoxicity may result in diabetic ketoacidosis, hyperosmolar hyperglycaemic syndrome, dehydration, shock and other emergencies. Therefore, the assessment of $\beta$-cell function and insulin sensitivity were usually performed at 3 months, when PG control was stable, instead of the baseline. Therefore, in the present study, the amelioration of $\beta$-cell function was compared between the sitagliptin combined with metformin and insulin groups and no significant differences in HOMA-IR, HOMA- $\beta$, AUC-Ins, AUC-PG, AUC-PG/AUC-Ins, Ip/I0, $\Delta \mathrm{I} 60 / \Delta \mathrm{G} 60$, $\mathrm{DI}$ and $\mathrm{MBCI}$ were identified. Likewise, concomitant with the glucose-lowering effects of treatment, no differences in $\beta$-cell function or insulin resistance indexes were observed between the two groups, suggesting that early sitagliptin combined with metformin therapy in patients with newly diagnosed T2DM with severe hyperglycaemia leads to similar outcomes in terms of the recovery of $\beta$-cell function to those of insulin treatment. The present results regarding the effects of sitagliptin combined with metformin treatment on $\beta$-cell function were largely consistent with those from large trials investigating sitagliptin as an add-on therapy agent and mixed studies involving multiple trials $(28,29)$. 


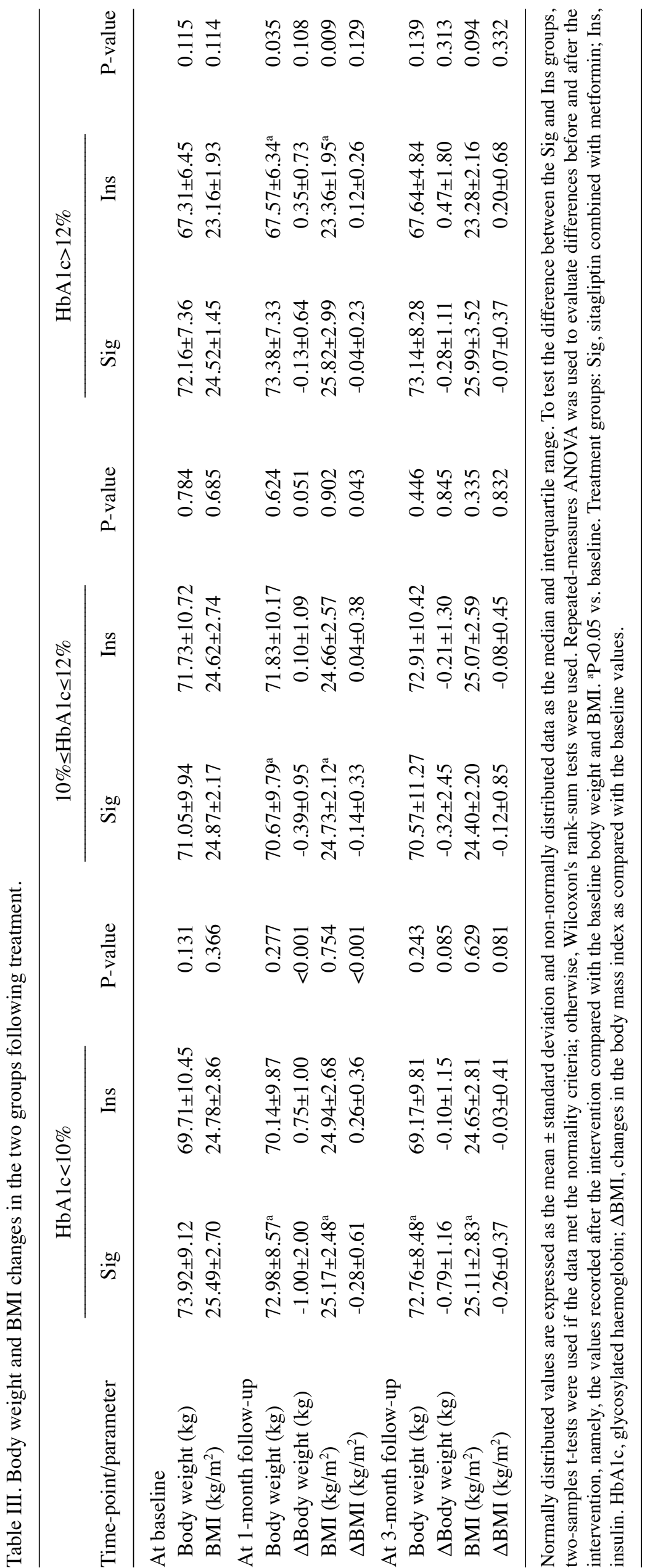


Table IV. Hypoglycaemia events in the two groups following treatment.

\begin{tabular}{lccc}
\hline Item & $\begin{array}{c}\text { Sig } \\
(\mathrm{n}=64)\end{array}$ & $\begin{array}{c}\text { Ins } \\
(\mathrm{n}=104)\end{array}$ & P-value \\
\hline Hypoglycaemic subjects, \% & 6.25 & 6.73 & 0.903 \\
HbA1c $<10 \%, \%$ & 6.90 & 9.09 & 0.773 \\
$10 \% \leq$ HbA1c $\leq 12 \%, \%$ & 3.70 & 5.45 & 0.729 \\
HbA1c $>12 \%, \%$ & 1.25 & 7.41 & 0.651 \\
\hline
\end{tabular}

Treatment groups: Sig, sitagliptin combined with metformin; Ins, insulin; HbA1c, glycosylated haemoglobin. Differences among groups were analysed using the $\chi^{2}$ test for nominal parameters.

Of note, trials involving sitagliptin for non-alcoholic fatty liver disease (NAFLD) have yielded promising results. NAFLD may deteriorate abnormal glucose and lipid metabolism, as well as increase potential risks of cardiovascular, cerebrovascular and peripheral vascular events for patients with T2DM (30). Iwasaki et al (31) demonstrated the efficacy of sitagliptin in patients with NAFLD and T2DM, with not only the parameters of diabetes improving, but also those of liver tests, following treatment with sitagliptin. Recent international guidelines universally emphasize the need for an individualized stepwise approach to pharmacotherapy for the management of T2DM and the choice of pharmacotherapy depends on numerous factors, including patient attributes and drug characteristics (e.g., route of administration, drug-drug interactions, safety profile and cost). As part of the present study, the cost of sitagliptin combined with metformin and insulin therapy was calculated and compared and no significant difference was observed in the cost of these two treatments (results not shown). In addition, in a real-world study (32), insulin therapy was associated with an increased risk of adverse events, such as a high rate of severe hypoglycaemia, glycaemic variability and weight gain, while sitagliptin was generally well tolerated, with most adverse events being of mild to moderate intensity and relatively few patients discontinuing treatment due to these events. Safety concerns have been raised regarding the potential risk of rare cases of pancreatitis and pancreatic cancer following the long-term use of DPP-4 inhibitors. However, no causal link between sitagliptin and these events has been established to date. With its convenient once-daily oral regimen, low potential for pharmacokinetic drug-drug interactions, as well as good efficacy and safety profiles, sitagliptin remains an important option for the management of patients with T2D.

Moreover, sitagliptin is approved for use in combination with insulin. Sitagliptin improves postprandial glycaemic control by stabilizing the active forms of endogenous incretins that are released following the ingestion of a meal, and thus, it is well suited as a supplement to insulin therapy. A comparison of benefits and drawbacks between sitagliptin combined with insulin and metformin combined with insulin is provided in Table SIII. Based on previous research, sitagliptin is able to stimulate the sarcolemmal translocation of the glucose transporter- 4 , in detriment of the fatty acyl translocase/CD36, and

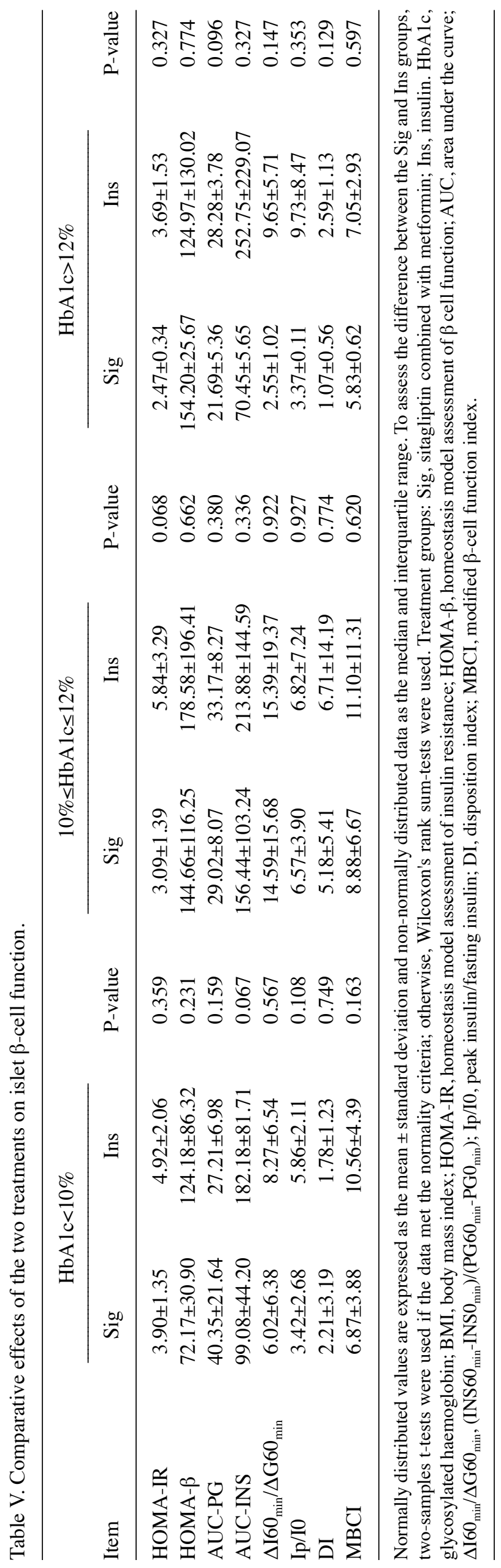


thus improve hyperglycemia, insulin resistance and GLP-1 levels (33). In addition, combining insulin with sitagliptin improved glycemic control and ameliorated oxidative stress and inflammation and thus induced significantly greater glucose-lowering effects than either drug alone (34).

Increases in body weight associated with specific antidiabetic agents are an undesired side effect in patients with T2DM. As indicated in previous studies, sitagliptin is thought to have a neutral effect on body weight, whereas insulin is associated with a slight weight gain in overweight and obese individuals with T2DM (35). However, the present study indicated that sitagliptin combined with metformin was associated with a considerable improvement in glycaemic control and slight weight reduction within the 1- and 3-month follow-up periods. A possible reason for this may be the role of metformin in weight loss and another may be the sitagliptin treatment-induced increase in GLP-1 levels. However, the underlying mechanisms of weight reduction by sitagliptin and their clinical relevance require to be further examined.

In the present study, both treatment regimens featured relatively low risks of hypoglycaemia. In the sitagliptin group, sitagliptin combined with metformin produced glucose-lowering effects that occurred in a glucose-dependent manner, indicating a low risk of hypoglycaemia. In the insulin group, insulin requirements were able to be estimated based on weight, with typical doses of 0.4-1.0 U/ $/ \mathrm{kg} / \mathrm{day}$, according to the 2019 ADA recommendations (7). In the present study, low-dose insulin was used at a total dosage of 0.1-0.4 U/kg/day and resulted in a low risk of hypoglycaemia.

One strength of the present study was that it was performed in a real-world setting. Sitagliptin combined with metformin was well-tolerated, significantly improved hyperglycaemia in a clinically challenging population with severe hyperglycaemia and led to a recovered $\beta$-cell function as compared with insulin treatment. However, there were several limitations to the present study. First, the follow-up duration was only 3 months. The longer-term effects of sitagliptin combined with metformin treatment on $\beta$-cell function warrant further investigation. Furthermore, the present study was a small-sample study, partly due to the uncertain safety of oral antidiabetic drugs in patients with glucotoxicity. Further studies with larger samples are required. In addition, each patient was given an SMBG record book and hypoglycaemic events were documented by the patients themselves, so there may be omissions. Another possible drawback was that, although there was no gender bias in the prevalence of T2DM, most participants were males. This gender imbalance may reflect better health care utilization by females (36), leading to reduced outpatient department use due to severe hyperglycaemia. A similar prevalence of male patients was also observed in other studies on severe hyperglycaemia (37).

In conclusion, the present results suggested that sitagliptin combined with metformin is a well-tolerated and effective treatment for improving early glycaemic excursions and $\beta$-cell function, with reduced hypoglycaemia and no weight gain. These results confirmed the efficacy and safety of sitagliptin combined with metformin in patients with newly diagnosed T2DM, suggesting that this combination is also beneficial as a first-line treatment in this patient population. Further larger and longer-term clinical studies are required to confirm the present results.

The results of the present and previous studies suggested that future guidelines regarding initiation therapy in newly diagnosed patients with T2DM and severe hyperglycaemia should also include non-insulin alternatives and a patient-centred approach should be used to guide the choice of pharmacological agents.

\section{Acknowledgements}

Not applicable.

\section{Funding}

This study was funded by the Award of the First Affiliated Hospital of Xi'an Jiaotong University, China (grant no. 2013YK20) and the Clinical Research Award of the First Affiliated Hospital of Xi'an JiaoTong University (Xi'an, China; grant no. XJTU1AF-CRF-2016-016).

\section{Availability of data and materials}

The datasets used and/or analysed during the present study are available from the corresponding author on reasonable request.

\section{Authors' contributions}

JS and BS conceived the study and participated in its design. $\mathrm{MD}, \mathrm{PF}, \mathrm{YW}, \mathrm{XZ}$ and $\mathrm{YH}$ collected the questionnaires and performed the statistical analyses. JS and MH confirmed the authenticity of the raw data and drafted the manuscript. JS, MH and JW interpreted the data. All authors have read and approved the final manuscript.

\section{Ethics approval and consent to participate}

This study was approved by the Ethics Committee of the First Affiliated Hospital of Xi'an JiaoTong University (Xi'an, China; no. XJTU1AF2016LSL-048). Informed consent was obtained from all participants. This study was registered with ClinicalTrials.gov under no. NCT03180281.

\section{Patient consent for publication}

Not applicable.

\section{Competing interests}

The authors declare that they have no competing interests.

\section{References}

1. Pratley RE and Weyer C: The role of impaired early insulin secretion in the pathogenesis of type II diabetes mellitus. Diabetologia 44: 929, 2001.

2. American Diabetes Association: 15. Diabetes care in the hospital: Standards of medical care in diabetes-2020. Diabetes Care 43 (Suppl 1): S193-S202, 2020.

3. Haak T: Initial combination with linagliptin and metformin in newly diagnosed type 2 diabetes and severe hyperglycemia. Adv Ther 29: 1005-1015, 2012. 
4. American Diabetes Association: 5. Lifestyle management: Standards of medical care in diabetes-2019. Diabetes Care 42 (Suppl 1): S46-S60, 2019.

5. Garber AJ, Abrahamson MJ, Barzilay JI, Blonde L, Bloomgarden ZT, Bush MA, Dagogo-Jack S, DeFronzo RA, Einhorn D, Fonseca VA, et al: Consensus statement by the American association of clinical endocrinologists and American college of endocrinology on the comprehensive type 2 diabetes management algorithm-2017 executive summary. Endocr Pract 23: 207-238, 2017.

6. American Diabetes Association Approaches to glycemic treatment. Sec. 7. In standards of medical care in diabetes-2016. Diabetes Care 39 (Suppl 1): S52-S59, 2016.

7. American Diabetes Association: 9. Pharmacologic approaches to glycemic treatment: Standards of medical care in diabetes-2019. Diabetes Care 42 (Suppl 1): S90-S102, 2019.

8. Pastores SM: ACP journal club. Review: Intensive insulin therapy does not reduce mortality but increases severe hypoglycemia in hospitalized patients. Ann Intern Med 155: JC1-JC12, 2011

9. Nyenwe E: Intensive insulin therapy in hospitalised patients increases the risk of hypoglycaemia and has no effect on mortality, infection risk or length of stay. Evid Based Med 17: 8-9, 2012

10. Aleali AM, Payami SP, Latifi SM, Yazdanpanah L, Hesam S and Khajeddin N: Evaluation of psychological resistance to insulin treatment in type II diabetic patients. Diabetes Metab Syndr 12: 929-932, 2018

11. Sanchez-Rangel E and Inzucchi SE: Metformin: Clinical use in type 2 diabetes. Diabetologia 60: 1586-1593, 2017.

12. Zinman B, Aroda VR, Buse JB, Cariou B, Harris SB, Hoff ST, Pedersen KB, Tarp-Johansen MJ and Araki E; PIONEER 8 Investigators: Efficacy, safety, and tolerability of oral semaglutide versus placebo added to insulin with or without metformin in patients with type 2 diabetes: The PIONEER 8 trial. Diabetes Care 42: 2262-2271, 2019.

13. Scott R, Morgan J, Zimmer Z, Lam RLH, O'Neill EA Kaufman KD, Engel SS and Raji A: A randomized clinical trial of the efficacy and safety of sitagliptin compared with dapagliflozin in patients with type 2 diabetes mellitus and mild renal insufficiency: The CompoSIT-R study. Diabetes Obes Metab 20: 2876-2884, 2018.

14. Thornberry NA and Gallwitz B: Mechanism of action of inhibitors of dipeptidyl-peptidase-4 (DPP-4). Best Pract Res Clin Endocrinol Metab 23: 479-486, 2009.

15. Ahrén B, Landin-Olsson M, Jansson PA, Svensson M, Holmes D and Schweizer A: Inhibition of dipeptidyl peptidase-4 reduces glycemia, sustains insulin levels, and reduces glucagon levels in type 2 diabetes. J Clin Endocrinol Metab 89: 2078-2084, 2004.

16. Dicker D: DPP-4 inhibitors: Impact on glycemic control and cardiovascular risk factors. Diabetes Care 34 (Suppl 2): S276-S278, 2011.

17. American Diabetes Association: Standards of medical care in diabetes-2013. Diabetes Care 36 (Suppl 1): S11-S66, 2013.

18. Matthews DR, Hosker JP, Rudenski AS, Naylor BA, Treacher DF and Turner RC: Homeostasis model assessment: Insulin resistance and beta-cell function from fasting plasma glucose and insulin concentrations in man. Diabetologia 28: 412-419, 1985.

19. Burden RL and Faires JD: Numerical analysis. J R Statist Soc 71: $48-50,2005$

20. Li Q, Wang L, Xiao L, Wang Z, Wang F, Yu X, Yan S and Wang Y: Effect of intensive insulin therapy on first-phase insulin secretion in newly diagnosed type 2 diabetic patients with a family history of the disease. Exp Ther Med 9: 612-618, 2015.

21. Li G, Yang W and Jiang Y: The possibility of (FINSxFPG)/(PG2h+PG1h-2FPG) being taken as an index of pancreatic $\beta$ cell insulin secretion in a population based study. Chin J Intern Med 13: 191-196, 2000.

22. Kang X, Wang C, Chen D, Lv L, Liu G, Xiao J, Yang Y, He L, Chen L, Li X, et al: Contributions of basal glucose and postprandial glucose concentrations to hemoglobin A1c in the newly diagnosed patients with type 2 diabetes-the preliminary study. Diabetes Technol Ther 17: 445-448, 2015.
23. Weir GC, Marselli L, Marchetti P, Katsuta H, Jung MH and Bonner-Weir S: Towards better understanding of the contributions of overwork and glucotoxicity to the beta-cell inadequacy of type 2 diabetes. Diabetes Obes Metab 11 (Suppl 4): 82-90, 2009.

24. Defronzo RA, Eldor R and Abdul-Ghani M: Pathophysiologic approach to therapy in patients with newly diagnosed type 2 diabetes. Diabetes Care 36 (Suppl 2): S127-S138, 2013.

25. Lyu X, Zhu X, Zhao B, Du L, Chen D, Wang C, Liu G and Ran X: Effects of dipeptidyl peptidase-4 inhibitors on beta-cell function and insulin resistance in type 2 diabetes: Meta-analysis of randomized controlled trials. Sci Rep 7: 44865, 2017.

26. Cnop M, Vidal J, Hull RL, Utzschneider KM, Carr DB, Schraw T, Scherer PE, Boyko EJ, Fujimoto WY and Kahn SE: Progressive loss of beta-cell function leads to worsening glucose tolerance in first-degree relatives of subjects with type 2 diabetes. Diabetes Care 30: 677-382, 2007.

27. Mu J, Petrov A, Eiermann GJ, Woods J, Zhou YP, Li Z Zycband E, Feng Y, Zhu L, Roy RS, et al: Inhibition of DPP-4 with sitagliptin improves glycemic control and restores islet cell mass and function in a rodent model of type 2 diabetes. Eur $\mathbf{J}$ Pharmacol 623: 148-154, 2009.

28. Heise T, Larbig M, Patel S, Seck T, Hehnke U, Woerle HJ and Dugi K: The dipeptidyl peptidase-4 inhibitor linagliptin lowers postprandial glucose and improves measures of $\beta$-cell function in type 2 diabetes. Diabetes Obes Metab 16: 1036-1039, 2014

29. Leibowitz G, Cahn A, Bhatt DL, Hirshberg B, Mosenzon O, Wei C, Jermendy G, Sheu WH, Sendon JL, Im K, et al: Impact of treatment with saxagliptin on glycaemic stability and $\beta$-cell function in the SAVOR-TIMI 53 study. Diabetes Obes Metab 17: 487-494, 2015

30. Tarantino G, Citro V and Capone D: Nonalcoholic fatty liver disease: A challenge from mechanisms to therapy. J Clin Med 9: $15,2019$.

31. Iwasaki T, Yoneda M, Inamori M, Shirakawa J, Higurashi $T$, Maeda S, Terauchi Y and Nakajima A: Sitagliptin as a novel treatment agent for non-alcoholic fatty liver disease patients with type 2 diabetes mellitus. Hepatogastroenterology 58: 2103-2105, 2011.

32. Zhou FL, Ye F, Berhanu P, Gupta VE, Gupta RA, Sung J, Westerbacka J, Bailey TS and Blonde L: Real-world evidence concerning clinical and economic outcomes of switching to insulin glargine $300 \mathrm{U} / \mathrm{ml}$ vs other basal insulins in patients with type 2 diabetes using basal insulin. Diabetes Obes Metab 20: 1293-1297, 2018.

33. Kelany ME, Hakami TM, Omar AH and Abdallah MA: Combination of sitagliptin and insulin against type 2 diabetes mellitus with neuropathy in rats: Neuroprotection and role of oxidative and inflammation stress. Pharmacology 98: 242-250, 2016.

34. Ramírez E, Picatoste B, González-Bris A, Oteo M, Cruz F, Caro-Vadillo A, Egido J, Tuñón J, Morcillo MA and Lorenzo Ó: Sitagliptin improved glucose assimilation in detriment of fatty-acid utilization in experimental type-II diabetes: Role of GLP-1 isoforms in Glut4 receptor trafficking. Cardiovasc Diabetol 17: 12, 2018

35. American Diabetes Association: 4. Lifestyle management: Standards of medical care in diabetes-2018. Diabetes Care 41 (Suppl 1): S38-S50, 2018.

36. Bertakis KD, Azari R, Helms LJ, Callahan EJ and Robbins JA: Gender differences in the utilization of health care services. J Fam Pract 49: 147-452, 2000.

37. Babu A, Mehta A, Guerrero P, Chen Z, Meyer PM, Koh CK, Roberts R, Schaider J and Fogelfeld L: Safe and simple emergency department discharge therapy for patients with type 2 diabetes mellitus and severe hyperglycemia. Endocr Pract 15: 696-704, 2009.

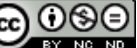

This work is licensed under a Creative Commons Attribution-NonCommercial-NoDerivatives 4.0 International (CC BY-NC-ND 4.0) License. 